\title{
Inhaltsübersicht über Band 7
}

\author{
Judentum und Christentum
}

1 Christentum und nichtchristliche Religionen - die Erklärung

Papst Pauls VI. anläßlich des 2. Vatikanischen Konzils

1.1 Der Text der Erklärung in Auszügen

1.2 Ein Gespräch mit dem Weihbischof von Hildesheim, Heinrich Pachowiak

1.3 Stellungnahmen beim 81. Deutschen Katholikentag 1966

1.3.1 Ernst Ludwig Ehrlich (Basel)

1.3.2 Gertrud Luckner (Freiburg/Br.)

2 Pinchas Lapide: „Warum sollte ein Jude Papst Pius XII. verteidigen?“

3 Tantur - Ein ökumenisches Institut für theologische Studien auf dem Wege nach Bethlehem

4 Juden und Christen beim 83. Deutschen Katholikentag

4.1 Gemeinschaftsgottesdienst

4.2 Arbeitskreis „Die Gemeinde und die jüdischen Mitbürger“

4.3 Das Referat von Willehad Paul Eckert

5 Gemeinsames Vaterunser für Juden und Christen? - Das christlichjüdische Problem beim Ökumenischen Pfingsttreffen in Augsburg

6 Der evangelische Kirchentag 1973 in Düsseldorf

7 „Christen und Juden“. Eine Studie des Rates der Evangelischen Kirche in Deutschland

8 Verleihung des Romano Guardini-Preises der Katholischen Akademie in Bayern am 9. März 1976 an Teddy Kollek und Professor Talmon

9 Gespräch mit Josef Burg über Judentum und Zionismus

10 "Juden und Christen“ - Die Ansprache des evangelischen Bischofs von Berlin, Martin Kruse, in der Kaiser-Wilhelm-Gedächtniskirche

11 Pnina Navè Levinson auf dem 85. Deutschen Katholikentag 1978 in Freiburg 
12 Achtzig Jahre Erlöserkirche in Jerusalem

13 Juden und Christen im neuen Katechismus - Ein Gespräch mit Wilhelm Breuning

14 Die Rede Philipp Jenningers bei einer Feierstunde der Gesellschaft für christlich-jüdische Zusammenarbeit

15 Die Verleihung der Buber-Rosenzweig-Medaille an Heinz Kremers

16 „Woche der Brüderlichkeit“ in der Rhein-Ruhr-Halle Duisburg

17 Die christlich-jüdischen Veranstaltungen beim 89. Deutschen Katholikentag in Aachen vom 10.-14. September 1986

18 „Suchet der Stadt Bestes - Brüderlichkeit in der modernen Gesellschaft“: Eröffnung der Woche der Brüderlichkeit in Berlin

19 Otto-Hirsch-Medaille für Pfarrer Fritz Majer-Leonhard

20 Okumene auf den Stationen Trier, Jerusalem und Frankfurt Pater Laurentius Klein berichtet über seine Erfahrungen

21 Die zweite Pastoralreise Johannes Pauls II. in die Bundesrepublik

21.1 Die Seligsprechung der Karmeliterin Edith Stein

21.2 Begegnung mit dem Zentralrat der Juden in Deutschland 21.2.1 Die Ansprache von Werner Nachmann 21.2.2 Die Ansprache des Papstes

21.3 Die Seligsprechung des Jesuitenpaters Rupert Mayer in München

22 Der evangelische Kirchentag 1987 in Frankfurt

22.1 "Seht, welch ein Mensch“ - Oberrabiner Albert Friedlander spricht in der Katharinenkirche in Frankfurt

22.2 Shalom Ben Chorin zum Auferstehungsbericht im JohannesEvangelium

23 Bundeskanzler Helmut Kohl überreicht den Sternberg-Preis des Internationalen Rates der Christen und Juden an Gertrud Luckner

24 Heinz M. Bleicher zum 65. Geburtstag 


\section{Juden und Judentum in der Bundesrepublik}

1 Vom "Jüdischen Gemeindeblatt“ für die britische Zone zur „Allgemeinen Jüdischen Wochenzeitung“
1.1 „Wir, die deutschen Juden“ - Karl Marx im „Jüdischen Gemeindeblatt“
1.2 „Vor einer schweren Aufgabe“ - Eine Broschüre zu Karl Marx’ 60. Geburtstag
1.3 Zum Tod von Karl Marx
1.4 Hermann Lewy, neuer Chefredakteur der „Allgemeinen Jüdischen Wochenzeitung“"

2 Axel Springer stiftet Glockenturm und Geläut für die Jerusalems-Kirche in Berlin

3 Fünfundzwanzigjähriges Jubiläum der Jüdischen Gemeinde in Berlin

4 Werner Nachmann: „Die jüdische Gemeinschaft in der Bundesrepublik Deutschland seit $1945^{“}$

5 Die „bibliotheca judaica“ des Lothar Stiehm Verlages in Heidelberg

6 "Jüdische Maler im Berlin der Jahrhundertwende“: Eine Ausstellung, organisiert von der Gesellschaft für christlich-jüdische Zusammenarbeit

7 Die Stiftung Volkswagenwerk fördert Forschungsprojekte zur Emigration deutschsprachiger Wissenschaftler 1933-1945 und zum Thema "Juden und Judentum in der Volkskultur"

8 „Erinnern bedeutet nicht dauernd mahnen und bemäkeln“ - Ein Interview der „Welt“ mit Pinchas Lapide

9 "Juden in Deutschland - 40 Jahre nach dem Holocaust“: Ein Vortrag Ernst Cramers vor der israelisch-deutschen Gesellschaft in Tel Aviv

10 Glückwünsche zum jüdischen Neujahrsfest

10.1 Grußwort des Bundespräsidenten

10.2 Grußwort des Bundeskanzlers

10.3 Grußworte der Parteien

11 Einwanderungshilfe nach Israel: Ein Gespräch mit dem Vertreter der Jewish Agency, Uri Aloni 
12 Das Salomon-Ludwig-Steinheim-Institut für deutsch-jüdische Geschichte an der Universität/Gesamthochschule Duisburg

13 Mündliche Anfrage der Grünen im Landtag zur Erhaltung und Restaurierung von Synagogen in Rheinland-Pfalz

14 Geburtstagsempfang für Heinz Galinski im Großen Saal der Jüdischen Gemeinde in Berlin

15 Die Einweihung der neuen Synagoge in Freiburg/Br.

16 Der Tod von Hans Rosenthal

17 „In schwieriger Mission“- Yohanan Meroz über seine Zeit als Botschafter Israels in der Bundesrepublik

18 Heinz Galinski wird Ehrenbürger von Berlin

18.1 Die Laudatio des Regierenden Bürgermeisters von Berlin, Eberhard Diepgen

18.2 Die Danksagung Heinz Galinskis

19 Der Friedrich Hänssler Verlag

20 Eine Ausstellung zur Geschichte des Philanthropin in Frankfurt

21 Die Synagoge von Ahrweiler

21.1 Auszüge aus einer Veröffentlichung von Superintendent Warnecke

21.2 Ein Gespräch mit Superintendent Warnecke

22 Internationaler Jüdischer Kongreß für Medizin und Halacha vom 16.-20. November 1988 in Berlin

22.1 Die Eröffnungsansprache Heinz Galinskis

22.2 Auszüge aus der Rede der Bundesministerin für Jugend, Familie, Frauen und Gesundheit, Rita Süssmuth

22.3 Grußwort des Senators für Gesundheit und Soziales, Ulf Fink

22.4 Der Vorsitzende der Jüdischen Ärzte und Psychologen in Berlin, Roman M. Skoblo

22.5 Rolf Winau: „Der jüdische Beitrag zur deutschen Medizin“ 


\section{Antisemitismus und Neonazismus in der Bundesrepublik}

1 Verfassungsfeinde von rechts - Ein Kommentar Hendrik van Berghs zum Jahresbericht des Verfassungsschutzes 1964

2 Hendrik van Bergh: „Das Geheimnis der Organisation ,Spinne‘. Gibt es eine geheime Fluchthilfe-Organisation für ehemalige Nationalsozialisten?“

3 „Erfahrung aus der Beobachtung und Abwehr rechtsradikaler und antisemitischer Tendenzen im Jahre 1965“: Ein Bericht des Bundesinnenministers

4 Der deutsche Botschafter in Israel, Rolf Pauls, zu den Erfolgen der NPD bei den bayerischen Landtagswahlen

4.1 Die Erklärung für die israelische Presse

4.2 Interview mit der israelischen Zeitung „Davar“

5 Fünf-Punkte-Erklärung der Bundesregierung zum Links- und Rechtsradikalismus

6 Synagogenschändung in München

7 „Die Verantwortung aller demokratischen Parteien gegenüber Anfängen antisemitischer Tendenzen": Aktuelle Stunde des Deutschen Bundestages

8 Renate Köcher, Institut für Demoskopie Allensbach: „Deutsche und Juden vier Jahrzehnte danach." Eine Repräsentativbefragung im Auftrag des ,Stern' im Jahre 1986

9 „Ausmaß und Formen des heutigen Antisemitismus":

Eine Studie des Instituts für Demoskopie Allensbach im Jahre 1988 


\section{Inhaltsübersicht über Band 8}

\section{Austausch in Kunst und Wissenschaft}

1 Die Anfänge des kulturellen Austausches zwischen der Bundesrepublik und Israel

2 Die Auswirkungen des Eichmann-Prozesses auf die kulturellen Beziehungen

3 Die Entwicklung der wissenschaftlichen Zusammenarbeit zwischen Israel und der Bundesrepublik

4 „Honorary Fellowship“ der Hebräischen Universität Jerusalem für Ludwig Erhard

5 Die Stiftung Volkswagenwerk richtet Forschungslehrstuhl im WeizmannInstitut ein

6 Die Arbeit Axel Springers und der Axel-Springer-Stiftung für Israel

6.1 Das Israel-Museum in Jerusalem

6.2 Eine Rede Axel Springers im Leo-Baeck-Institut in Jerusalem

6.3 Stiftung des Ottilie-Springer-Lehrstuhls in Waltham

6.4 Die Einweihung der Bibliothek des Israel-Museums

6.5 Verleihung der Ehrendoktorwürde der Bar-Han-Universität an Axel Springer

6.6 Die Axel-Springer-Stiftung unterstützt den Bau eines Rehabilitationszentrums in Jerusalem

6.7 Axel Springer erhält die Ehrendoktorwürde der Hebräischen Universität Jerusalem

6.8 Das Leo-Baeck-Institut ehrt Axel Springer

7 Die Stiftung Volkswagenwerk

7.1 Schwerpunkte

7.2 Gründung

7.3 Kuratorium

7.4 Zusammenarbeit mit Israel

8 Ein Gespräch mit Herbert Wehner anläßlich seiner Ernennung zum Ehrendoktor der Hebräischen Universität Jerusalem 


\section{Inhaltsübersicht über Band 8}

9 Erste kulturpolitische Gespräche auf Direktorenebene in Jerusalem Ein Gespräch mit Ministerialdirektor Lahn

10 Für die Errichtung des Max-Born-Lehrstuhles für Naturphilosophie stellt die Stiftung Volkswagenwerk 1 Million DM zur Verfügung

11 „Plakate aus Israel“: Eine Ausstellung in Berlin

12 Reaktionen auf den Tod Axel Springers

12.1 Die Trauerfeier

12.2 Kondolenzen

12.3 Heinz Galinski: „Axel Springer, ein unersetzlicher Gesprächspartner"

13 Niels Hansen: Vierzig Jahre Staat Israel - Eindrücke als deutscher Botschafter 1981-1985

14 Aus der Dankrede von Ministerpräsident Johannes Rau anläßlich der Verleihung der Ehrendoktorwürde der Universität Haifa am 2. April 1986

15 Der israelische Premierminister Shimon Peres spricht vor Journalisten in Berlin

16 Deutsch-israelische Zusammenarbeit im Bereich der beruflichen Bildung, des Wissenschaftsaustausches, des Studentenaustausches und des Jugendaustausches. Ergebnis einer Reise des Parlamentarischen Staatssekretärs beim Bundesminister für Bildung und Wissenschaft, Anton Pfeifer.

17 Die deutsch-israelische Wissenschaftsstiftung nimmt ihre Arbeit auf

18 Das 12. deutsch-israelische Lehrerseminar in Jerusalem

19 Deutsch-israelische Gemeinschaftsprojekte, gefördert von der Stiftung Volkswagenwerk

19.1 Notationsmöglichkeiten für elektronische Musik

19.2 Spieltheorie in der ökologischen Forschung

19.3 Feste Teilchen in strömenden Gasen oder Flüssigkeiten

19.4 Zur Rezeption jüdischer Geschichte im modernen Israel

19.5 Beitrag zum schadstoffarmen Ottomotor

19.6 Zur Diagnostik von Verbrennungsprozessen

19.7 Optische Untersuchungsmethoden zur Halbleiterforschung

19.8 Zur Verbrennung von Dieselkraftstoff 
20 Eine Darstellung des Bundesministeriums für Forschung und Technologie zur wissenschaftlich-technologischen Zusammenarbeit mit Israel

21 "Jettchen Geberts Kinder“ - Eine Ausstellung des Leo-Baeck-Instituts in Bonn

22 Die Fritz-Naphtali-Stiftung in Israel

23 Abkommen zwischen der Bundesrepublik und Israel über die Errichtung einer Stiftung für wissenschaftliche Forschung und Entwicklung

23.1 Der Wortlaut des Abkommens

23.2 Zur Arbeit des Kuratoriums

24 Neue historische Reihe der Universität Tel Aviv beim Bleicher-Verlag: Ein Gespräch mit Heinz Bleicher

25 Ein fotographisches Porträt von Israel

26 „Die Palästinenserin“: Diskussion im Westdeutschen Fernsehen über die Inszenierung von Peter Eschberg

27 Das israelische Philharmonische Orchester in Bonn

28 Europatagung der Freundesgesellschaften der Hebräischen Universität Jerusalem in Frankfurt

29 Honorary Fellowship an die Vizepräsidentin des Deutschen Bundestages, Annemarie Renger, und an den Staatsminister Lutz Stavenhagen verliehen

30 Die Zusammenarbeit der Fritz Thyssen Stiftung mit Israel

30.1 Aus dem Jahresbericht 1975/76

30.2 Aus dem Jahresbericht 1977/78

30.3 Aus dem Jahresbericht 1978/79

30.4 Aus dem Jahresbericht 1979/80

30.5 Aus dem Jahresbericht 1980/81

30.6 Aus dem Jahresbericht 1981/82

30.7 Aus dem Jahresbericht 1982/83

30.8 Aus dem Jahresbericht 1983/84

30.9 Aus dem Jahresbericht 1984/85

30.10 Aus dem Jahresbericht 1985/86

30.11 Aus dem Jahresbericht 1986/87

31 Daimler Benz unterstützt Projekte in Israel

31.1 Zusammenstellung der geförderten wissenschaftlichen Projekte 1973-1987 
31.2 Konzertreise des Bundesjugendorchesters 1986 nach Israel 31.2.1 Ein Gespräch mit Rita Süssmuth

31.2.2 Ein Gespräch mit Dr. Gottschalk von Daimler Benz

31.3 Der Gottlieb-Schumacher-Lehrstuhl an der Universität Haifa

31.3.1 Historischer Hintergrund

31.3.2 Die Ansprachen bei der Einweihung

31.3.3 Ein Gespräch mit Alex Carmel über die Bedeutung des Lehrstuhls

31.3.4 Finanzielle Beteiligung des Landes Baden-Württemberg

32 The International Center for Peace in the Middle East

33 Friedrich-Ebert-Stiftung: 20 Jahre Friedensarbeit für Israel

34 Die Jerusalem Foundation - Ein Gespräch mit Ministerpräsident Bernhard Vogel am 26.2.1988

35 Eine Briefmarkenausstellung im Siegburger Rathaus zum 40jährigen Jubiläum des Staates Israel

36 Deutsch als Wahlfach an Israels Schulen

37 Statistische Übersicht über Humboldt-Stipendiaten aus Israel

38 Städtepartnerschaften zwischen der Bundesrepublik und Israel

39 Das Forschungszentrum Ben Gurion in Sde Boker - Ein Gespräch mit dem Leiter, Asher Ben Natan

40 Neue deutsch-israelische Kulturkonsultationen

40.1 Interview mit Ministerialdirektor Witte, Leiter der Kulturabteilung im Auswärtigen Amt

40.2 Der Text des Abkommens

\section{Jugendaustausch}

1 Entstehung und Entwicklung des deutsch-israelischen Jugendaustausches

2 Deutsche Jugendgruppen 1964 in Israel

3 Die Gesellschaft für christlich-jüdische Zusammenarbeit veranstaltet 1965 eine Israelreise mit zwei Jugendgruppen 
4 Über 450 Lehrer und Schüler aus Köln besuchten Israel zwischen 1960 und 1965

5 Ein Interview mit Bruno Heck, Bundesminister für Jugend, Familie und Gesundheit, über den Jugendaustausch mit Israel

6 Richtlinien für den Jugendaustausch zwischen der Bundesrepublik Deutschland und Israel

7 Erste offizielle Schülergruppe aus Israel in der Bundesrepublik

8 Weiterer Ausbau des deutsch-israelischen Jugendaustausches

9 Konferenz über Fragen des Jugendaustausches in Israel

10 Zehn Jahre Jugendaustausch Köln - Tel Aviv

11 Tagungen der gemischten Fachkommission für den deutsch-israelischen Jugendaustausch

11.1 Die 1. Tagung in Tel Aviv 1969

11.2 Die 3. Tagung im Mai 1971

11.3 Israelische Kritik am Programm des Jugendaustausches

12 Neuer Start im Jahre 1972

13 „Sonderregelung für die Förderung von Programmen des deutschisraelischen Jugendaustausches in der Bundesrepublik Deutschland“ Erlaß des Bundesministers für Jugend, Familie und Gesundheit für das Haushaltsjahr 1972

14 Nach dem Yom-Kippur-Krieg: Die Jugendarbeit mit Israel beginnt wieder

15 Kritik von deutschen Verbänden

16 Gemeinsame Bestimmungen für die Durchführung und Förderung des Jugendaustausches vom Gemischten Fachausschuß

17 Die internationale Jugendarbeit der Evangelischen Kirche Deutschlands

18 Die überarbeitete Fassung der Grundlagen des deutsch-israelischen Jugendaustausches

19 "Jugend und Jugendarbeit in Israel. Versuch einer Darstellung von Hermann Sieben“: Auszüge aus einer Broschüre des IJAB 
Inhaltsübersicht über Band 8

20 Auszüge aus dem Bericht des Bundesministers für Jugend, Familie und Gesundheit über Maßnahmen der internationalen Jugendarbeit

21 Israelische Jugendliche in der Bundesrepublik Deutschland 1986

21.1 Israelische Schüler in Bonn

21.2 Gymnasiasten aus Kiriat Chaim in Mannheim

21.3 Junge Israelis in Freiburg

22 Einweihung der Jugendbegegnungsstätte in Auschwitz

23 „Bewältigung der Zukunft - Erinnerung an die Vergangenheit - Zukunft für unsere Jugend“": Der Vortrag von Vizepräsident Westphal bei der Frankfurter Loge von B'nai-B'rith am 24.2.1987

24 Tagung des Gemischten Fachausschusses für den deutsch-israelischen Jugendaustausch

\section{Namensregister}

